\title{
Mapping Electronic Orbitals in Real Space
}

\author{
Stefan Löffler ${ }^{1,2,3}$ \\ ${ }^{1}$ Department of Materials Science and Engineering, McMaster University, Hamilton, Canada. \\ ${ }^{2}$ University Service Centre for Transmission Electron Microscopy, Vienna University of Technology, \\ Vienna, Austria. \\ ${ }^{3}$ Institute of Solid State Physics, Vienna University of Technology, Vienna, Austria.
}

Electronic states are the key to most material properties including mechanical stability, chemical bonding, electrical conductivity, magnetism and optical properties. Because of their paramount importance across many fields, their direct mapping has attracted much attention in recent years. Using elastic electron scattering, total charge densities could be measured on the nanometer scale [1,2]. On the surface, molecular orbitals could be resolved using STM [3]. But the direct mapping of individual electronic states in real space in the bulk phase - the key to materials design - has been elusive so far.

In this work, an overview is given over recent developments of mapping individual, specific electronic orbitals using transmission electron microscopy (TEM) and electron energy loss spectrometry (EELS). In TEM, it is possible to focus the probe beam to sub-Ångström spatial resolution using a state-of-the-art aberration corrected instrument. By sending a focused electron probe through the sample, energy and momentum can be exchanged between the probe electrons and the sample electrons, giving rise to a unique energy and momentum distribution of the probe beam after the sample which can be measured. By selecting an appropriate energy range using a slit aperture, specific transitions to individual states in the sample can be selected [4], and by scanning the probe beam over the sample, information about the probability distribution of those states in real space can be deduced. Fig. 1 shows an example of an orbital map of the $\mathrm{e}_{\mathrm{g}}$ contributions to Rutile.

To interpret the experimental data, a thorough understanding of the image formation mechanism and extensive computer simulations are indispensable. In the particular case of combining TEM and EELS, both elastic and inelastic scattering effects of the probe beam inside the specimen need to be considered [5]. The elastic scattering effects are usually taken into account using either the multislice approach or the Bloch wave approach. For modelling the inelastic scattering, a very versatile tool is the mixed dynamic form factor (MDFF) in the density matrix framework. Only recently, a novel approach to diagonalize the MDFF was developed [6]. It not only allows to combine realistic predictions of the electronic orbitals in the material (e.g., from density functional theory calculations) with the inelastic scattering calculation with reasonable computational effort, but also provides physical insight into the underlying scattering processes. One crucial result of this theoretical and numerical treatment is that the point-group symmetry of the atom under investigation plays a decisive role in the shape of the resulting maps. Only if the symmetry is low enough (as is the case in Rutile, but also near defects, interfaces, etc.), a bonding direction can be imaged.

By combining simulations and experiments, it is thus possible to map orbital information in the bulk in real space using state-of-the-art TEMs. This will pave the way for completely new possibilities and materials design, in particular in the vicinity of defects and interfaces [7]. 
References:

[1] JM Zuo et al, Nature 401 (1999), p. 49.

[2] JC Meyer et al, Nature Materials 10 (2011), p. 209.

[3] J Repp et al, Science 312 (2006), p. 1196.

[4] W Hetaba et al, Micron 63 (2014), p. 15.

[5] MP Prange et al, Physical Review Letters 109 (2012), p. 246101.

[6] S Löffler, V Motsch and P Schattschneider, Ultramicroscopy 131 (2013), p. 39.

[7] The author acknowledges financial support by the Austrian Science Fund (FWF) under grant number I543-N20 and SFB F45 FOXSI. The experimental data in Fig. 1 was kindly provided by Matthieu Bugnet, Nicolas Gauquelin, Sorin Lazar, and Gianluigi Botton. The orbital visualization was kindly provided by Elias Assmann and Karsten Held.

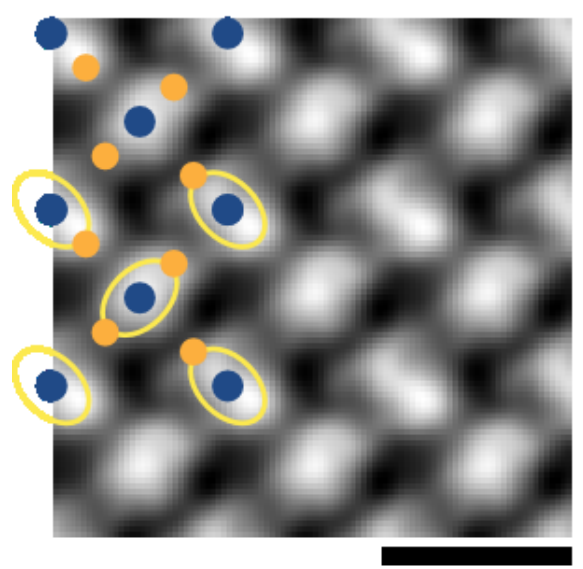

$5 \AA$

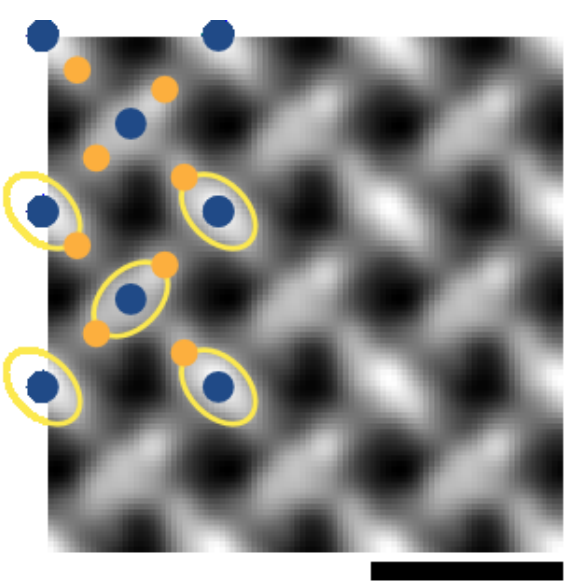

5 A

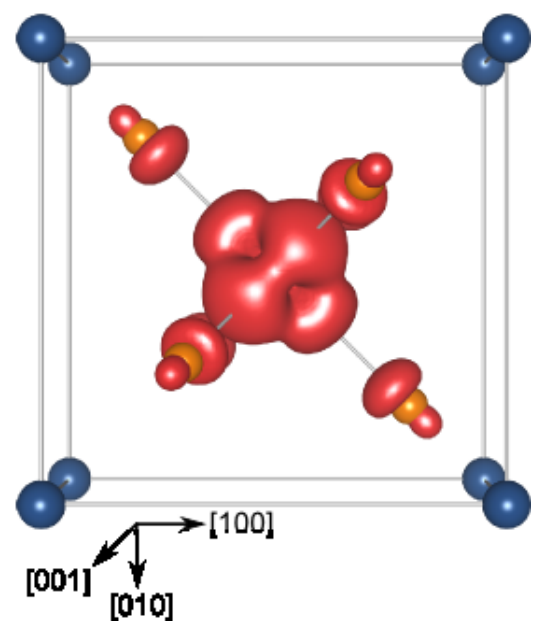

Fig. 1: Gaussian-filtered experimental (left) and simulated (center) map of the $\mathrm{e}_{\mathrm{g}}$ orbital contributions to the Ti $\mathrm{L}_{2,3}$ edge in $20 \mathrm{~nm}$ thick Rutile in [001] projection using an $80 \mathrm{keV}$ probe beam. The overlays show the position of the Ti atoms (blue) and of the $\mathrm{O}$ atoms (orange). The ellipses (yellow) are guides for the eye only. Both images were replicated in a $3 \times 3$ rater for better visibility. The right panel shows the summed three-dimensional charge density of the $\mathrm{e}_{\mathrm{g}}$ Wannier functions around the central Ti atom. 